\title{
Evaluación de la velocidad del LCR en pacientes con neuroinfección y RM en contraste de fase
}

\section{Velocity evaluation of CSF flow in patients with neuroinfection by}

\section{phase-contrast MRI}

Rocío C. Saldaña-Sánchez ${ }^{1 *}, M^{a}$ del Carmen Amezcua-Herrera², Alejandro E. Vega-Gutiérrez²,

Roberto Ramírez-López ${ }^{2}$ y Christian Ramos-Peñafiel ${ }^{4}$

${ }^{1}$ Servicio de Alta Especialidad; ${ }^{2}$ Servicio de Resonancia Magnética; ${ }^{3}$ Servicio de Imagen Diagnóstica; ${ }^{4}$ Servicio de Medicina Interna y Hematología, Hospital General de México "Dr. Eduardo Liceaga", Ciudad de México, México

\section{RESUMEN}

Introducción: La resonancia magnética en contraste de fase permite estudiar la dinámica del líquido cefalorraquídeo(LCR) demanera cualitativa y cuantitativa. Material y métodos: Se realizó un estudio retrospectivo y descriptivo con una muestra de 25 estudios, de los cuales 13 se efectuaron en pacientes sanos y 12 tenían datos de neuroinfección, hasta un 91.7\% secundario a tuberculosis meníngea. Resultados: La media de velocidad del LCR en pacientes con neuroinfección fue de $2.549 \mathrm{~cm} / \mathrm{s}(0.573-5.200)$ en comparación con los individuos sanos con media de $2.882 \mathrm{~cm} / \mathrm{s}$ (1.0-6.420). Conclusiones: Al analizar las diferencias de medias en $\mathrm{cm} / \mathrm{s}$ no se identificó diferencia significativa $(\mathrm{p}=0.614)$ con 95\% de intervalo de confianza.

Palabras clave: Resonancia magnética en contraste de fase. Líquido cefalorraquídeo. Neuroinfección. Tuberculosis meníngea.

\section{ABSTRACT}

Introduction: Contrast phase MR allows qualitative and quantitative study of cerebrospinal fluid. Material and methods: This study was a retrospective and descriptive, we had a sample of 25 patients, 13 studies were in healthy patients and 12 studies with neuroinfection in the last group $91.7 \%$ was secondary to TB meningitis. Results: The average velocity in neuroinfection cases is $2.549 \mathrm{~cm} / \mathrm{s}(0.573$ a 5.200$)$, compared with the healthy 
patients with average velocity $2.882 \mathrm{~cm} / \mathrm{s}(1.0$ a 6.420). Conclusion: There is not significant differences between the healthy patients compare with neuroinfection cases.

Key words: Phase contrast magnetic resonance. Cerebrospinal flow magnetic resonance. Neuroinfection. Meningeal Tuberculosis.

\section{INTRODUCCIÓN}

El líquido cefalorraquídeo (LCR), llamado también líquido cerebroespinal, es una sustancia clara e incolora que protege al encéfalo y la médula espinal del daño físico y químico. También transporta oxígeno y glucosa desde la sangre hasta las neuronas y neuroglia. El LCR circula de forma continua a través del espacio "subaracnoideo", en los planos cerebral y espinal; este espacio se encuentra entre las meninges aracnoides y piamadre ${ }^{1}$.

La capacidad total de la cavidad cerebroespinal en el adulto es de 1,600 a 1,700 ml. El volumen del LCR no es constante, sino que sufre variaciones por diferentes motivos y puede reducirse por cambios fisiológicos en la hiperventilación ${ }^{2}$.

El flujo del LCR discurre de los ventrículos laterales al tercero por el agujero de Monro y al cuarto por el acueducto de Silvio; de allí puede salir por el agujero de Magendie (medial) o agujero de Lushka (lateral) hacia la cisterna magna ${ }^{3}$. Este movimiento tiene lugar de manera pulsátil impulsado por la sístole cardíaca, la inhalación respiratoria y, en menor proporción, el movimiento de los cilios de las células ependimarias. La intensidad de estos pulsos de LCR forma un gradiente rostrocaudal: en promedio $60 \%$ menor a nivel lumbar respecto de los ventrículos, lo que facilita el desplazamiento del líquido ${ }^{4}$.
Los plexos coroideos (PC) producen sobre todo el LCR y el $10 \%$ a 30\% del líquido procede del líquido intersticial cerebral. La secreción del LCR resulta de un bombeo constante de $\mathrm{Na}^{3}$.

La secreción del LCR varía en forma circadiana, pero se produce la mayor cantidad en las primeras horas de la mañana. Pueden inhibirla los esteroides, acetazolamida, diuréticos y temperaturas bajas ${ }^{4}$. El LCR se reabsorbe en especial en los senos venosos y vellosidades aracnoideas. Su tasa máxima de reabsorción es de $1.5 \mathrm{ml}$ / min; en el canal espinal, la reabsorción puede ocurrir a 0.11 a $0.23 \mathrm{ml} / \mathrm{min}$, la que aumenta con el ejercicio. En los senos venosos, sobre todo el superior, su reabsorción tiene lugar en forma pasiva, dependiente del gradiente de presión del espacio subaracnoideo y de los propios senos (diferencial de presión de 5-7 $\mathrm{mmHg}^{5}$.

La aracnoiditis basal es un proceso inflamatorio meníngeo y del espacio subaracnoideo que produce engrosamiento de éstos, así como adhesiones de la duramadre que causan complicaciones, entre ellas aracnoiditis adhesiva, neuropatías, mielopatías y radiculopatías ${ }^{6}$.

La aracnoiditis puede ser secundaria a múltiples causas:

- Inflamatoria no infecciosa: es efecto de operaciones, hemorragia subaracnoidea y administración de fármacos intratecales, como anestésicos, medios de contraste y esteroides. 
- Neoplásica: como metástasis de cáncer de mama, cáncer pulmonar, melanoma, linfoma no Hodgkin, glioblastoma, ependimoma y cáncer de plexos coroideos.

- Infecciosa: viral, bacteriana, fúngica y parasitaria.

La infección del sistema nervioso central por Mycobacterium tuberculosis es la forma sistémica más peligrosa y puede afectar al encéfalo, médula espinal, nervios craneales, espinales y meninges debido a la elevada tasa de complicaciones y secuelas neurológicas.

Sólo $2 \%$ a $5 \%$ de todos los pacientes con tuberculosis (TB) sistémica desarrollan esta complicación, pero el porcentaje puede aumentar hasta $10 \%$ en sujetos con VIH. La neurotuberculosis puede aparecer a cualquier edad; en los países subdesarrollados, los niños también pueden mostrar esta complicación ${ }^{7}$.

La principal manifestación de la TB en el sistema nervioso puede consistir en meningitis, tuberculomas cerebrales y espondilitis tuberculosa $^{8,9}$. Tanto la tomografía computarizada (TC) como la resonancia magnética contrastada son útiles en el diagnóstico de las complicaciones de la meningitis, aunque la resonancia magnética se considera una mejor herramienta.

Las características de imagen de la neuroinfección por TB consisten en realce anormal leptomeníngeo que se extiende hacia el espacio subaracnoideo del sulcus, las cisternas basales (cisterna prepontina, ambiens, supraselar), tentorio, hoz cerebral, ventriculomegalia relacionada en ocasiones con hidrocefalia, infartos periventriculares y tuberculomas, y relace en el tentorio. Una revisión retrospectiva de la tomografía en hallazgos de 289 pacientes con tuberculosis reveló que el $88 \%$ tenía anormalidades de imagen con hidrocefalia hasta en $75 \%$, realce parenquimatoso en $40 \%$, infartos cerebrales en $20 \%$, realce de las cisternas basales en $30 \%$ y tuberculomas un $10 \%{ }^{10,11}$.

La aracnoiditis optoquiasmática cursa con pérdida de la visión. La resonancia magnética en contraste de fase es un método que permite cuantificar y estudiar la dirección del flujo del LCR, incluso en flujos bajos como el del acueducto de Silvio donde el flujo es regular, el sistema ventricular, los espacios subaracnoideos y la médula espinal, según los estudios de Sherman de 1986, Barkhof de 1994 y Jacobson de 1996. Las primeras anomalías estudiadas con este método de imagen fueron las de Chiari y los quistes aracnoideos de Barkhof en 1994 y Menick en 2001. Es un método empleado en la práctica posquirúrgica, por ejemplo en las ventriculostomías del tercer ventrículo y en el flujo del LCR en la acueductoplastia endoscópica que realizó Schroeder en el año $2000^{12}$.

En el estudio de Unal, et al. de 2009 se evaluó a una población de 60 pacientes sanos y se descartaron trastornos obstructivos del sistema ventricular. Se dividió a los enfermos en cinco grupos de edad, se encontró una velocidad máxima en pacientes normales de 1.41 y $11.6 \mathrm{~cm} / \mathrm{s}$ y no se identificaron diferencias significativas en los valores de velocidades entre los distintos grupos de edad (los pacientes de 14 años tenían velocidades $>11.6 \mathrm{~cm} / \mathrm{s})$. Se estudió el área del acueducto de Silvio con valores de 1.2 a $4.8 \mathrm{~mm}^{2}$ (media, 2.6). Cuatro de los casos mostraron un área 
$<1.5 \mathrm{~mm}$, sin diferencias significativas en los parámetros de flujo del $\mathrm{LCR}^{13}$.

Según el trabajo de Flórez, et al., la cisterna prepontina es un espacio con complejidad anatómica que es difícil segmentar para extraer los diferentes parámetros. En su conocimiento no existían valores publicados de flujo de LCR obtenidos en esta región. Mediante el método presentado fue posible delinear la cisterna pontina al eliminar los pixeles de la arteria basilar y calcular el volumen por ciclo $^{14}$.

El estudio de Tawfik, et al. publicó el caso de un grupo de pacientes sin comorbilidades, con velocidades máximas de 3.5 a $7.7 \mathrm{~cm} / \mathrm{s}$ y un volumen de 26.6 a $24.7 \mu \mathrm{L} / \mathrm{ciclo}^{15}$.

En el estudio de Forner, et al., que incluyó a un total de 108 sujetos, 61 sujetos sanos fueron controles y 47 pacientes tenían enfermedad adjunta; 19 de ellos se clasificaron en el grupo de pacientes con enfermedad cerebrovascular isquémica (ECI) y 28 pacientes en el grupo de hidrocefalia a presión normal (HPN). Los pacientes del grupo sano registraron una velocidad máxima sistólica de $32 \mathrm{~mm} / \mathrm{s}$, velocidad máxima diastólica de $35 \mathrm{~mm} / \mathrm{s}$ y un volumen por ciclo de 44 microlitros $^{16}$.

Cheng publicó en el año 2012 los modelos de flujo del LCR en pacientes con aracnoiditis con una permeabilidad de 105, 107 y 109 m² $^{2}$ durante el ciclo cardiaco y demostró que la presión caudal de la aracnoiditis no se ve afectada por los cambios de permeabilidad, la presión se eleva en relación con el incremento de la aracnoiditis y se reduce la permeabilidad. La diferencia de presión se incrementa aún más con el descenso de la permeabilidad; en esta etapa, el flujo del LCR casi es inexistente en el espacio subaracnoideo y el flujo se dirige hacia la superficie ventral del espacio subaracnoideo $^{17}$.

No se cuenta en la actualidad con algún estudio por RM en contraste de fase que demuestre la alteración de la dinámica del LCR de forma cuantitativa en pacientes con aracnoiditis basal.

Mediante este estudio se intenta determinar el porcentaje de estudios con datos de aracnoiditis basal que cursan con alteración del flujo del líquido cefalorraquídeo, además de evaluar la RM en contraste de fase como método diagnóstico no invasivo en la cuantificación del LCR en el acueducto de Silvio, en relación con estudio sin comorbilidades.

La RM en contraste de fase detecta de forma cuantitativa la velocidad del flujo del líquido cefalorraquídeo en el acueducto de Silvio, en pacientes sanos y con neuroinfección; un objetivo fue también establecer una relación entre el área del acueducto de Silvio con la velocidad del LCR y determinar si la pleocitosis de éste produce alteración en la dinámica del flujo del LCR, realizado de forma no invasiva, sin necesidad de aplicar contraste endovenoso, radiación y preparación previa; el propósito es obtener mayor información sobre este método de imagen en esta afección, ya que no se cuenta con estudios previos de otros autores.

\section{MATERIAL Y MÉTODOS}

Se realizó un metaanálisis retrospectivo, cuantitativo y descriptivo de un total de 25 


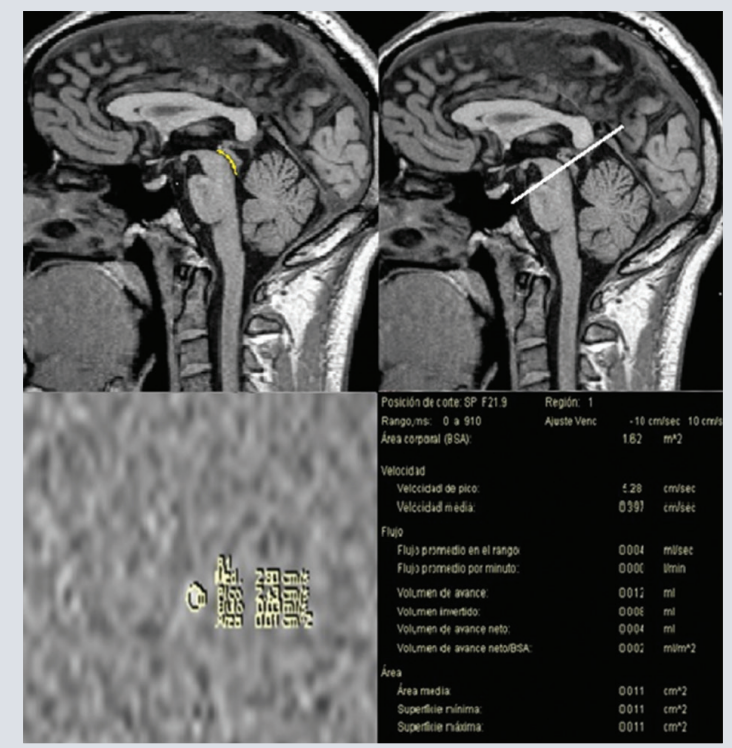

Figura 1. Planeación del sitio de medición del acueducto de Silvio (línea amarilla) en adquisición sagital con angulación de $15^{\circ}$ colocada en el tercio medio del acueducto. Luego de la planeación se observa la adquisición axial del acueducto de Silvio con colocación del ROI en su periferia y obtención de los resultados.

estudios con resonancia y contraste de fase, de los cuales 12 se realizaron en pacientes con neuroinfección y 13 en individuos sanos; el intervalo de edad fue de 18 a 80 años y las técnicas se efectuaron entre las 8 y 11 horas debido a la variación de la velocidad en relación con el ciclo circadiano del LCR.

La adquisición de datos partió de estudios de resonancia magnética de cráneo obtenidos en un escáner de $1.5 \mathrm{~T}$ (Siemens) en pacientes sanos y con neuroinfección; el estudio cuantitativo produjo imágenes axiales 2D-FISP (100TR/16TE, ángulo $15^{\circ}$ ), con grosor de corte de $4 \mathrm{~mm}$ situado perpendicular al acueducto (matriz de 512 x 512 y FOV de 160) y con velocidad codificada de $200 \mathrm{~mm} / \mathrm{s}$. A partir de estas imágenes se determinaron la velocidad

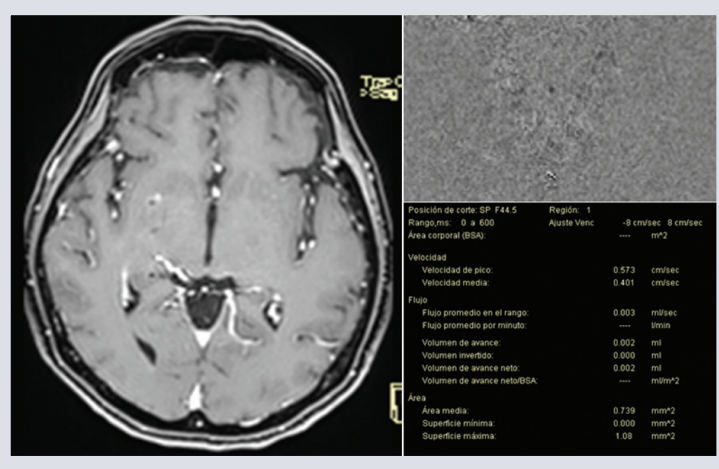

Figura 2. Paciente con datos de neuroinfección con tuberculoma en el núcleo lenticular derecho, con adquisición axial en secuencia en contraste de fase, proceso de la imagen y obtención de resultados con disminución de la velocidad en relación con los pacientes sanos.

media, la velocidad máxima, el flujo y el volumen del líquido que pasa por el acueducto durante la sístole y la diástole cardíaca (Fig. 1).

\section{RESULTADOS}

Se estudió a un total de 25 pacientes con diagnóstico de neuroinfección, atendidos en la Unidad 207 en el servicio de resonancia magnética del Hospital General de México "Dr. Eduardo Liceaga" en el lapso de enero del 2018 a octubre del 2019, durante el cual se realizó resonancia magnética convencional con secuencia en contraste de fase.

El 91.7\% de los casos $(\mathrm{n}=11)$ tuvo diagnóstico de TB y el 8.3\% $(n=1)$ diagnóstico de criptococosis (Fig. 2). De manera global se analizó en su mayoría a pacientes del género masculino, 56\% $(\mathrm{n}=14)$ contra $44 \%(\mathrm{n}=11)$ del género femenino. El género con mayor frecuencia de meningitis correspondió al masculino $(n=8)$ con $57.1 \%$. 


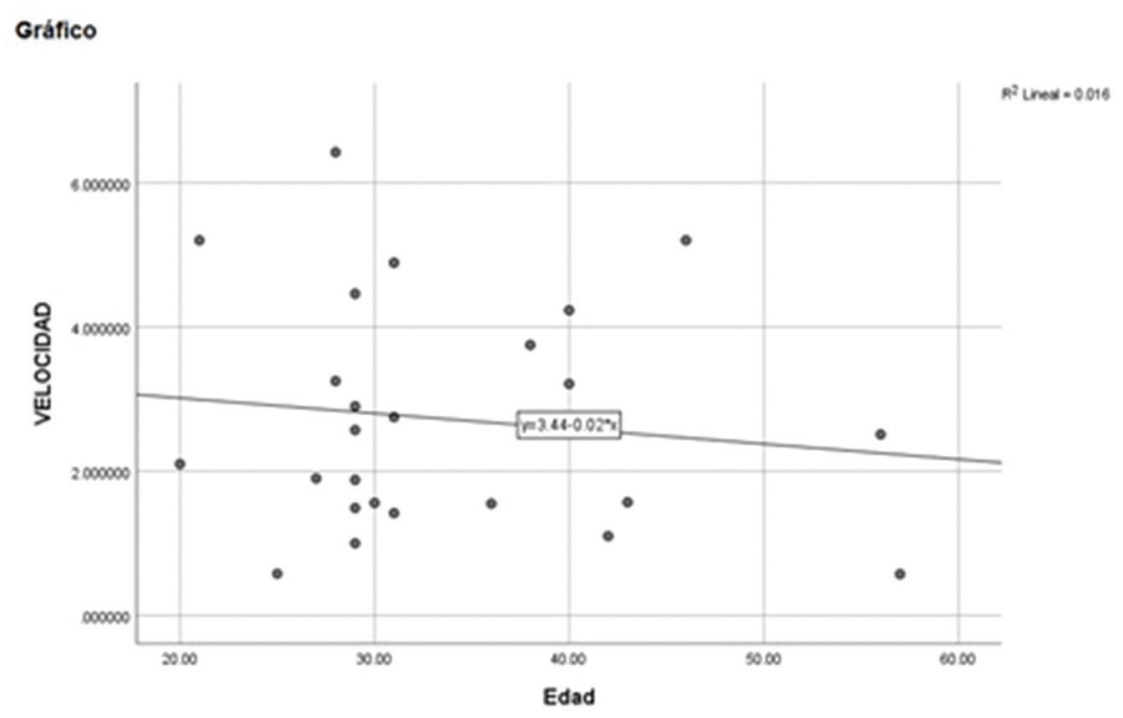

Figura 3. Velocidad y edad.

La media de edad de los pacientes con neuroinfección fue de 37.6 años (20-57 años), en comparación con los pacientes sanos que tuvieron una media de edad de 30.1 años (27-40 años). Esta diferencia de edad (30 años en pacientes control vs 37 años en pacientes con neuroinfección) es significativa con $p=0.45$ y $95 \%$ de intervalo de confianza.

La velocidad máxima del LCR posee una media total de $2.722 \mathrm{~cm} / \mathrm{s}$, con intervalo de 0.573 a 0.420 $\mathrm{cm} / \mathrm{s}$; en el análisis de casos en pacientes con neuroinfección, la media de velocidad fue de $2.549 \mathrm{~cm} / \mathrm{s}(0.573$ - 5.200) en comparación con los individuos sanos con media de $2.882 \mathrm{~cm} / \mathrm{s}(1.0$ - 6.420); al analizar las diferencias de medias en $\mathrm{cm} / \mathrm{s}$ no se identificó diferencia significativa ( $\mathrm{p}$ $=0.614$ ) con $95 \%$ de intervalo de confianza.

La diferencia de velocidad por género fue de $2.775 \mathrm{~cm} / \mathrm{s}$ para el género masculino (0.5736.420) y de $2.554 \mathrm{~cm} / \mathrm{s}$ para el femenino
(1-5.200 cm/s). Al analizar la diferencia de acuerdo con el género no se identificó diferencia significativa $(0.855 \mathrm{~cm} / \mathrm{s})$. Al intentar buscar una correlación entre la edad y la velocidad no se reconoció una correlación lineal, pero en los pacientes $>50$ años la media de velocidad fue menor en comparación con los $<50$ años (R2 lineal de 0.016) (Fig. 3).

$\mathrm{Al}$ analizar la correlación entre la pleocitosis de LCR con la velocidad media no se identificó una correlación directa (R2 de 0.001) (Fig. 4). La velocidad media fue de 0.6324 (0.04 - $2.29 \mathrm{~cm} / \mathrm{s}$ ) y al tratar de hallar una correlación entre la velocidad máxima y la velocidad media no se identificó una correlación directa, pero sí una tendencia ascendente de manera lineal entre la velocidad media y la máxima (R2 lineal de 0.036)

$\mathrm{Al}$ analizar la diferencia entre el flujo promedio y el volumen de avance no se reconoció 


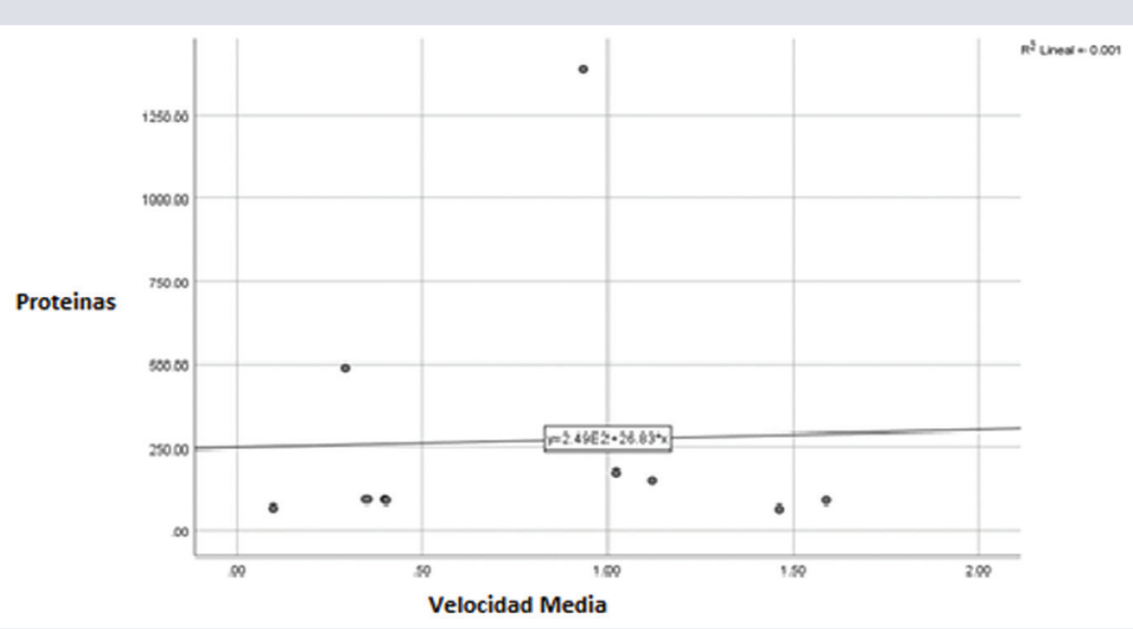

Figura 4. Velocidad y proteínas.

ninguna diferencia significativa entre las medias $(0.437$ con $95 \%$ de intervalo de confianza y 0.621 con intervalo de confianza al $95 \%$, respectivamente).

La media del acueducto de Silvio de forma global fue de $1.461 \mathrm{~mm}^{2}$, en límites de 0.206 y $3.93 \mathrm{~mm}^{2}$. En los pacientes sanos fue de $1.582\left(0.206-3.99 \mathrm{~mm}^{2}\right)$ en comparación con los enfermos de 1.330 (0.237 - 0.320). La diferencia de la media del acueducto de Silvio con los casos del grupo control no mostró diferencia significativa en el volumen $(\mathrm{P}=0.524, \mathrm{n}=95 \%)$.

$\mathrm{Al}$ analizar el patrón de distribución del área de Silvio contra la velocidad no se halló un patrón específico y se presentó como una sigma (R2 de 0.227) (Fig. 5).

\section{DISCUSIÓN}

Cheng publicó en el año 2012 modelos de flujo del LCR en pacientes con aracnoiditis con una permeabilidad de 105, 107 y 109 $\mathrm{m}^{2}$ durante el ciclo cardiaco, y demostró que la presión caudal de la aracnoiditis no se afecta por los cambios de permeabilidad y que la presión se eleva en relación con el incremento de la aracnoiditis, al tiempo que se reduce la permeabilidad. La diferencia de presión aumenta aún más con el descenso de la permeabilidad; en esta etapa, el flujo del LCR casi es inexistente en el espacio subaracnoideo y el flujo se redirige a la superficie ventral del espacio subaracnoideo.

La muestra pequeña realizada en este estudio es una limitante en la obtención de resultados de velocidad del líquido cefalorraquídeo; en este protocolo no se logró demostrar variación en la velocidad máxima del LCR en pacientes con neuroinfección respecto de los sujetos sanos. No existe una tendencia al aumento de la velocidad del LCR en relación con las dimensiones del acueducto de Silvio ni con el incremento de la pleocitosis. 


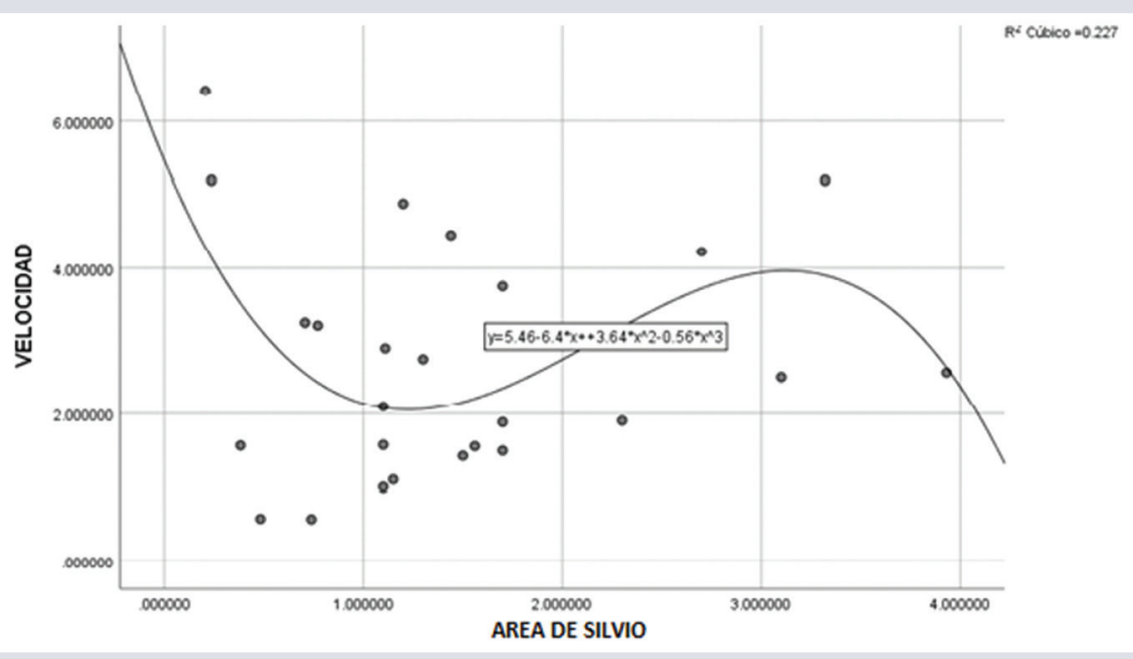

Figura 5. Velocidad y área de Silvio.

\section{CONCLUSIONES}

La velocidad máxima del LCR en individuos sanos muestra una media total de $2.882 \mathrm{~cm} / \mathrm{s}$ $(1.0-6.420 \mathrm{~cm} / \mathrm{s})$ y en pacientes con neuroinfección la media de velocidad fue de $2.549 \mathrm{~cm} / \mathrm{s}(0.573-5.200)$ por lo que no se observa una diferencia significativa entre ambos grupos.

En cuanto a la correlación entre la velocidad del líquido cefalorraquídeo y el área del acueducto de Silvio, no hay relación significativa al igual que la pleocitosis en relación con la velocidad del LCR.

\section{AGRADECIMIENTOS}

Los autores expresan su agradecimiento a sus profesores del Hospital General de México que hicieron posible compartir un año más de aprendizaje.

\section{FINANCIAMIENTO}

El estudio se basó en materiales existentes en el Hospital General de México.

\section{CONFLICTO DE INTERESES}

Este estudio no tiene ningún conflicto de intereses.

\section{RESPONSABILIDADES ÉTICAS}

Protección de personas y animales. Los autores declaran que para esta investigación no se han realizado experimentos en seres humanos ni en animales.

Confidencialidad de los datos. Los autores declaran que en este artículo no aparecen datos de pacientes. 


\section{Derecho a la privacidad y consentimiento informado. Los autores declaran que en este artículo no aparecen datos de pacientes.}

\section{BIBLIOGRAFÍA}

1. Afifi A, Bergman R. Neuroanatomía funcional. 2da. ed. México: McGrawHill, 2006:259-270.

2. Rangel-Castillo L, Robertson CS. Management of intracranial hypertension. Crit Care Clin. 2006;22:713-32.

3. Brodbelt A, Stoodley M. An anatomical and physiological basis for CSF pathway disorders. Atenas: Informa Healthcare, 2010:1-8.

4. Gomes JA, Bhardwaj A. Normal intracranial pressure physiology. Irani DN, editor. Cerebrosipnal fluid in clinical practice. Philadelphia: Elsevier, 2009.

5. Morrison BM. Physiology of cerebrospinal fluid secretion, recirculation, and resorption. Cerebrosipnal fluid in clinical practice. Philadelphia: Elsevier, 2009:1.

6. Khan MU. Adhesive archanoiditis in mixed connected tissue disease. MBJ case report. 2016. Medscape.

7. Sri Krishna CK, Sandip C. Acquired Chiari malformation secondary to tuberculous arachnoiditis of the lumbar spine. Nuerological Society of India. 2016;64:1066-1068.
8. Osborn A. Neuroradiología diagnóstica. Enfermedad del encéfalo y sus cubiertas. Capítulo 4. PP 673-684.

9. Martínez HR. Tuberculosis del sistema nervioso central: conceptos actuales. Revista Mexicana de Nuerociencia. 2010:1-8.

10. Botha H, Ackerman C, Candy S, Carr JA, Griffith-Richards S, Bateman KJ. Reliability and diagnostic performance of $\mathrm{CT}$ imaging criteria in the diagnosis of tuberculous meningitis. PLoS One. 2012;7:e38982.

11. Andronikou S, Wieselthaler N, Smith B, Douis H, Fieggen AG, van Toorn R, et al. Value of early follow-up CT in paediatric tuberculous meningitis. Pediatr Radiol. 2005;35:1092-9.

12. Oner Z, Sagir K. Quantitative evaluation of normal aqueductal cerebrospinal fluid flow using phase-contrast cine MRI according to age and sex. Anat Rec (Hoboken). 2017;300(3):549-555.

13. Özkan U. Cine phase-contrast MRI evaluation of normal aqueductal cerebrospinal fluid flow according to sex and age. Diagn Interv Radiol. 2009;15:227-231.

14. Flórez N. Acta Biol Colomb. 2010;15(3):213-220.

15. Tawfik. AMPhase-contrast MRI CSF flow measurements for the diagnosis of normal-pressure hydrocephalus: observer agreement of velocity versus volume parameters. AJR. 2017; 208:838-843.

16. Forner GJ. Quantitative phase-contrast MRI study of cerebrospinal fluid flow: a method for identifying patients with normal-pressure hydrocephalus. Neurología (English Edition). 2014;29(2):68-75.

17. Shaokoon C. The presence of arachnoiditis affects the characteristics of CSF flow in the spinal subarachnoid space: a modelling study. Journal of Biomechanics. 2012;45:1186-1191. 